\title{
Effect of Methylprednisolone Plus Azithromycin on Fractional Exhaled Nitric Oxide and Peripheral Blood Eosinophils in Children with Refractory Mycoplasma Pneumoniae Pneumonia
}

\author{
Hui Zhou, Xiaojuan Chen and Jing Li \\ Department of Pediatrics, Daqing Oilfield General Hospital, Deqing, Heilongjiang Province, China
}

\begin{abstract}
Objective: To investigate the efficacy and adverse reactions of methylprednisolone plus azithromycin in the treatment of children with refractory mycoplasma pneumoniae pneumonia (RMPP) and its effect on fractional exhaled nitric oxide (FeNO) and peripheral blood eosinophils (EOS).

Study Design: Comparative study.

Place and Duration of Study: Daqing Oilfield General Hospital, China, from June 2019 to January 2021.

Methodology: A total of 102 children with RMPP were randomly divided into Group A $(n=51)$ and Group B $(n=51)$ by the random number table. Group A was treated with azithromycin alone, and Group B was treated with methylprednisolone plus azithromycin. The efficacy, adverse reactions, FeNO and EOS levels were compared.

Results: The total effective rate of treatment in Group B was higher than that in Group A $(p=0.006)$. Time of cough disappearance, lung shadow disappearance, defervescence and hospitalisation in Group B was shorter than that in Group A $(p=0.033,0.008,0.004$ and $<0.001$, respectively). After treatment, the FeNO and EOS levels in Group B were lower than those in Group A (both $p<0.001$ ). Conclusion: The efficacy of methylprednisolone plus azithromycin in treatment of RMPP is better than azithromycin alone. The former can reduce the levels of FeNO and EOS more effectively than the latter.
\end{abstract}

Key Words: Refractory mycoplasma pneumoniae pneumonia (RMPP), Methylprednisolone, Azithromycin, Fractional exhaled nitric oxide (FeNO), Eosinophils (EOS).

How to cite this article: Zhou H, Chen X, Li J. Effect of Methylprednisolone Plus Azithromycin on Fractional Exhaled Nitric Oxide and Peripheral Blood Eosinophils in Children with Refractory Mycoplasma Pneumoniae Pneumonia. J Coll Physicians Surg Pak 2022; 32(01):33-36.

\section{INTRODUCTION}

At present, there is no unified definition of refractory mycoplasma pneumoniae pneumonia (RMPP). Most studies refer to the diagnostic criteria of RMPP, proposed by Tamura et al., namely children are treated with macrolide alone for more than one week without improvement in clinical manifestations such as fever, and with clinical symptomsorimaging manifestations that are progressively worsening. ${ }^{1}$ Children with RMPP usually show persistent high fever, spasmodic cough, and pulmonary imaging findings such as atelectasis, massive consolidation and even necrotic pneumonia, lung abscess. ${ }^{2}$ RMPP can involve nervous system, hematologic system, bone, joint, muscle and skin. Therefore, effective treatment should betaken as soon as possible.

Correspondence to: Jing Li, Department of Pediatrics, Daqing Oilfield General Hospital, Deqing, Heilongjiang Province, China

E-mail: ivzobu@163.com

Received: August 06, 2021; Revised: October 09, 2021;

Accepted: November 24, 2021

DOI: https://doi.org/10.29271/jcpsp.2022.01.33
Azithromycin is a macrolides antibiotic. By combining subunits in the nucleus of bacteria, azithromycin can effectively inhibit the process of mycoplasma pneumoniae peptide conversion, so as to achieve a good anti-bacterial effect. ${ }^{3}$ Methylprednisolone is glucocorticoids, and it can regulate immunity and inflammatory response by inhibiting lipid-mediated inflammatory cytokines, and thus reduce the damage of pathogenic bacteria to the body. ${ }^{4}$ Some studies have confirmed that in treatment of RMPP in children with azithromycin alone, its effect is relatively slow, and the clinical response is relatively poor. ${ }^{5}$ Therefore, methylprednisolone can be used in combination with azithromycin, which can give full play to the synergistic effect of medicines and increase the therapeutic effect. ${ }^{6}$

Although most studies have confirmed the efficacy of methylprednisolone plus azithromycin in treatment of children with RMPP, the results of safety reports are different. It has been pointed out that there is no significant difference in the adverse reactions between azithromycin plus glucocorticoid and azithromycin alone in the treatment of RMPP in children. ${ }^{7}$ Some studies suggest that azithromycin plus glucocorticoid does not increase the adverse reactions of children with RMPP. ${ }^{8}$ Another 
study indicates that high dose methylprednisolone plus azithromycin is effective in the treatment of RMPP, but the incidence of adverse reactions is higher than that of methylprednisolone plus azithromycin, and azithromycin alone. ${ }^{9}$ Studies have shown that the incidence of adverse reactions of methylprednisolone plus azithromycin in treatment of RMPP in children is significantly lower than that treated with azithromycin alone. ${ }^{10,11}$

Fractional exhaled nitric oxide (FeNO) is produced by NO synthase in respiratory epithelial cells, and there is a correlation between its value and the severity of airway inflammation. ${ }^{12}$ However, there are few reports on the effect of methylprednisolone plus azithromycin on FeNO in children with RMPP. Peripheral blood eosinophils (EOS) are an indicator for allergic diseases. ${ }^{13}$ One study has shown that mycoplasma pneumoniae (MP) infection can increase airway EOS inflammatory response, leading to the increase of FeNO level and the change of peripheral blood EOS level. ${ }^{14}$ However, there are few reports on the effect of methylprednisolone plus azithromycin on EOS in children with RMPP.

The objective of this study was to investigate the efficacy and adverse reactions of methylprednisolone plus azithromycin in treatment of children with RMPP and its effect on FeNO and peripheral blood EOS.

\section{METHODOLOGY}

This comparative study was approved by the Ethics Committee of Daqing Oilfield General Hospital, China, and conducted from June 2019 to January 2021. A total of 102 children with RMPP were randomly divided into Group A $(n=51)$ and Group $B(n=$ 51 ) by the random number table, according to the sequential order of visit to the hospital. Inclusion criteria were symptoms not relieved with a progressive trend after one week of regular treatment with macrolide (erythromycin) alone; conformed to the diagnostic criteria for RMPP, that is, after standard treatment with macrolide antibiotics for seven days or more, the clinical signs were aggravated, the fever continued $\left(\geq 38.5^{\circ} \mathrm{C}\right.$, the lung imaging manifestations were aggravated, and the children were clearly infected with mycoplasma pneumoniae; ${ }^{1,15}$ and child patient aged 5-13 years. Exclusion criteria were allergies or contraindications to azithromycin and methylprednisolone, recent use of glucocorticoid-related medicines, complicated with pulmonarytuberculosis, immunodeficientchildren, complicated with other systems or organ serious diseases, and detected with other pathogens during pneumonia.

All children were given routine treatment including maintaining electrolyte and acid-base balance, physical hypothermia, expectorant, antiasthma, oxygen inhalation and so on. Group A was treated with azithromycin alone, that is, azithromycin was given intravenously $10 \mathrm{mg} / \mathrm{Kg}$ per day for three days, then discontinuation for four days, and then treated with azithromycin dispersible tablets $10 \mathrm{mg} / \mathrm{Kg}$ per day for three days and discontinuation for four days. The above was one course of treatment, and three consecutive courses of treat- ment were conducted. Group B was treated with methylprednisolone plus azithromycin, that is, therapy of Group A was added with sodium methylprednisolone succinate intravenously, treated with $2 \mathrm{mg} / \mathrm{Kg}$ per day for five days, and then $1 \mathrm{mg} / \mathrm{Kg}$ per day for two days. The above was a course of treatment, and three consecutive courses of treatment were conducted.

After treatment, the clinical effects of the two groups were compared. The criteria of curative effect were: (i) Remarkably effective: the clinical symptoms and signs of the children disappeared, the vital signs returned to normal, and the chest imaging examination showed that the shadow disappeared; (ii) Effective: the clinical symptoms and signs of the children were improved, the vital signs recovered obviously, and the chest imaging examination showed that the shadow was absorbed; and (iii) ineffective: there was no significant change or aggravation of the above indexes. ${ }^{16}$ Overall response rate $=$ (number of remarkably effective cases + number of effective cases) / total number of cases $\times 100 \%$.

The time of cough relief, defervescence, lung rale disappearance, chestradiographyshadow disappearanceand hospitalisation were compared between the two groups. The changes of FeNO and EOS levels before and after treatment were also compared between the two groups. FeNO was detected by Nakulun exhalation hydrogen analyser (model: Sunvou-P100) produced by China Wuxi Shangwo Medical Electronics Co., Ltd. The unit of test results was parts per billion (ppb). Two $\mathrm{mL}$ fasting venous blood was extracted and used to detect EOS by automatic blood cell counter. The incidence of adverse reactions was compared between the two groups during treatment.

SPSS version 25.0 software was used to process data. Measurement data in accordance with normal distribution were expressed by mean $\pm S D$, and independent sample t-test was used. Count data were expressed by $n(\%)$, and Chi-square test was used. The significant difference was defined as $p<0.05$.

\section{RESULTS}

The age of 102 children was $5-13$ years, with an average of 8.30 \pm 0.82 years. In Group A, 21 cases ( $41.18 \%$ ) showed remarkably effective, 17 cases (33.33\%) showed effective, and 13 cases (25.49\%) were ineffective. A total of 38 cases $(74.51 \%)$ were effective. In Group B, 32 cases (62.75\%) showed remarkably effective, 16 cases ( $31.37 \%$ ) as effective, and 3 cases $(5.88 \%)$ were ineffective, so the total effective rate was 48 cases (94.12\%).

Total effective rate of treatment in Group B was higher than that in Group A ( $p=0.006)$. Time of cough disappearance, lung shadow disappearance, effervescence and hospitalisation in Group B was shorter than that in Group A $(p=0.033,0.008$, 0.004 and $<0.001$, respectively, Tablel).

Before treatment, there was no difference in the FeNO and EOS levels between the two groups ( $p=0.603$ and 0.276 , respectively, Table II). After treatment, the FeNO and EOS levels in Group B were lower than those in Group A (both $p<0.001$, Tablell). 
There was no significant difference in incidence of adverse reactions such as nausea and vomiting, skin allergic reactions, facial flushing and constipation between the two groups ( $p=0.373$, Table III).

Tablel: Comparison of clinical parameters.

\begin{tabular}{|l|c|c|c|}
\hline Parameter & $\begin{array}{c}\text { Group A } \\
(\mathbf{n = 5 1 )}\end{array}$ & $\begin{array}{c}\text { Group B } \\
(\mathbf{n}=5 \mathbf{5 1})\end{array}$ & p-value \\
\hline $\begin{array}{l}\text { Time of cough } \\
\text { disappearance (d) }\end{array}$ & $8.00 \pm 3.09$ & $6.67 \pm 3.12$ & 0.033 \\
\hline $\begin{array}{l}\text { Time of lung shadow } \\
\text { disappearance (d) }\end{array}$ & $12.00 \pm 4.58$ & $9.43 \pm 5.02$ & 0.008 \\
\hline Time of defervescence (d) & $11.06 \pm 3.55$ & $8.82 \pm 4.18$ & 0.004 \\
\hline Time of hospitalization (d) & $14.82 \pm 3.78$ & $11.39 \pm 5.31$ & $<0.001$ \\
\hline
\end{tabular}

Tablell: Comparison of FeNOandEOSlevels.

\begin{tabular}{|l|c|c|c|}
\hline Parameter & $\begin{array}{c}\text { Group A } \\
(\mathbf{n = 5 1 )}\end{array}$ & $\begin{array}{c}\text { Group B } \\
(\mathbf{n = 5 1 )}\end{array}$ & p-value \\
\hline FeNO before treatment $(\mathrm{ppb})$ & $356.70 \pm 28.26$ & $353.83 \pm 27.30$ & 0.603 \\
\hline FeNO after treatment $(\mathrm{ppb})$ & $149.08 \pm 10.43$ & $97.30 \pm 6.94$ & $<0.001$ \\
\hline EOS before treatment $\left(\times 10^{9} / \mathrm{L}\right)$ & $0.46 \pm 0.03$ & $0.45 \pm 0.04$ & 0.276 \\
\hline EOS after treatment $\left(\times 10^{9} / \mathrm{L}\right)$ & $0.37 \pm 0.03$ & $0.28 \pm 0.02$ & $<0.001$ \\
\hline
\end{tabular}

Table III: Comparison of incidence of adverse reactions.

\begin{tabular}{|l|c|c|c|}
\hline Adverse reactions & $\begin{array}{c}\text { Group A } \\
(\mathbf{n = 5 1 )}\end{array}$ & $\begin{array}{c}\text { Group B } \\
(\mathbf{n = 5 1 )}\end{array}$ & p-value \\
\hline Nausea and vomiting $[\mathrm{n}(\%)]$ & $3(5.88)$ & $1(1.96)$ & - \\
\hline Skin allergic reactions $[\mathrm{n}(\%)]$ & $2(3.92)$ & $2(3.92)$ & - \\
\hline Facial flushing $[\mathrm{n}(\%)]$ & $2(3.92)$ & $1(1.96)$ & - \\
\hline Constipation $[\mathrm{n}(\%)]$ & $1(1.96)$ & $1(1.96)$ & - \\
\hline Total $[\mathrm{n}(\%)]$ & $8(15.69)$ & $5(9.80)$ & 0.373 \\
\hline
\end{tabular}

\section{DISCUSSION}

In view of the suboptional tolerance and the low safety of tetracycline and quinolones antibiotics in children, macrolides are mostly used in treatment of RMPP in children. Azithromycin is the second-generation macrolides, which is a semi-synthetic 15-ring compound, derived from erythromycin, and it is safer than erythromycin. At present, the prognosis of some children is poor in treatment of RMPP with azithromycin alone. ${ }^{6}$

It has been pointed out that azithromycin plus methylprednisolone is more effective in treatment of RMPP than azithromycin alone. This study also confirmed the above conclusion. ${ }^{5}$

It is found that the cough disappearance time, lung shadow disappearance time, antipyretic time and hospitalisation time in children, treated with azithromycin plus methylprednisolone, were shorter than those treated with azithromycin alone; suggesting that azithromycin plus methylprednisolone can better improve the symptoms of children and promote the recovery of children as soon as possible. The above results of this study were basically consistent with a previous meta-analysis. ${ }^{7}$ It is speculated that azithromycin plus methylprednisolone can play a synergistic role in making up for each other's advantages, actively improve the symptoms and signs of children with RMPP, and promote the improvement of ventilation function and recovery in children with RMPP.

Some studies suggest that there is a correlation between FeNO level and airway EOS inflammation; and FeNO can be used to predict eosinophilic airway infammation in uncontrolled asthmatic patients. ${ }^{17}$ Some studies have also shown that FeNO is closely related to children mycoplasma pneumonia. ${ }^{18}$

The results of this study showed that there was no difference in the FeNO and EOS levels between the two groups before treatment; but after treatment, the FeNO and EOS levels in Group B were lower than those in Group A, indicating that mycoplasma pneumonia infection can increase the EOS inflammatory reaction of airway and the FeNO and EOS levels in children. Compared with azithromycin alone, the combination of methylprednisolone and azithromycin can reduce the FeNO and EOS levels more effectively and thus promote the recovery of children morequickly.

This study found that nausea and vomiting, skin allergic reactions, facial flushing and constipation were common adverse events in children with RMPP treated with azithromycin alone or plus methylprednisolone, and there was no significant difference in incidence of adverse reactions between the two groups. The results of this study were basically consistent with a previous meta-analysis, ${ }^{7}$ suggesting that azithromycin plus methylprednisolone is safe and does not increase adverse events.

The limitation of this study is that the efficacy and adverse events of different doses of methylprednisolone plus azithromycin in the treatment of RMPP, as well as the effect on FeNO and EOS in children with RMPP, were not discussed. Furtherstudies are needed to investigate questions above.

\section{CONCLUSION}

The efficacy of methylprednisolone plus azithromycin in treatment of RMPP is better than azithromycin alone. The former can reduce the levels of FeNO and EOS more effectively than the latter.

\section{ETHICALAPPROVAL:}

This study was approved by the Ethical Committee of Daqing Oilfield General Hospital, China.

\section{PATIENTS' CONSENT:}

The guardians of all subjects accepted the study with informed consent.

\section{CONFLICT OF INTEREST:}

The authors declared no conflict of interest.

\section{AUTHORS' CONTRIBUTION:}

HZ, XC: Data collection and analysis, research design and conception, manuscript writing, and result interpretation.

JL: Literature search, discussion and final approval.

$\mathrm{HZ}$ and XC contributed equally at all stages of the article. 


\section{REFERENCES}

1. Tamura A, Matsubara K, Tanaka T, Nigami H, Yura K, Fukaya T. Methylprednisolone pulse therapy for refractory Mycoplasma pneumoniae pneumonia in children. J Infect 2008; 57(3):223-8. doi:10.1016/j.jinf.2008.06.012.

2. Ling $X$, Sun $X$, Kong $H$, Peng S, Yu Z, Wen J , et al. Chinese herbal medicine for the treatment of children and adolescents with refractory mycoplasma pneumoniae pneumonia: A systematic review and a meta-analysis. Front Pharmacol 2021; 12:678631-46. doi: 10.3389/fphar. 2021.678631.

3. Oliver ME, Hinks TSC. Azithromycin in viral infections. Rev Med Virol 2021; 31(2):e2163-75. doi: 10.1002/ rmv.2163.

4. Ranjbar K, Moghadami M, Mirahmadizadeh A, Fallahi M J, Khaloo V, Shahriarirad R, et al. Methylprednisolone or dexamethasone, which one is superior corticosteroid in the treatment of hospitalised COVID-19 patients: A triple-blinded randomised controlled trial. BMC Infect Dis 2021; 21(1):337-44. doi:10.1186/s12879-02106045-3.

5. Luo Z, Luo J, Liu E, Xu X, Liu Y, Zeng F, et al. Effects of prednisolone on refractory Mycoplasma pneumoniae pneumonia in children. Pediatr Pulmonol 2014; 49(4): 377-80. doi: 10.1002/ppul.22752.

6. Shan L, Liu X, Kang X, Wang F, Han X, Shang Y. Effects of methylprednisolone or immunoglobulin when added to standard treatment with intravenous azithromycin for refractory Mycoplasma pneumoniae pneumonia in children. World J Pediatr 2017; 13(4): 321-7. doi: 10.1007/s12519-017-0014-9.

7. Qiu J, Huang L, Shao M, Chai Y, Zhang H, Li X, et al. Efficacy and safety of azithromycin combined with glucocorticoid on refractory Mycoplasma pneumoniae pneumonia in children: A PRISMA-compliant systematic review and meta-analysis. Medicine 2020; 99(22): e20121-8. doi: 10.1097/MD.0000000000020121.

8. Zheng MG. Effect analysis of short course of glucocorticoid adjuvant therapy on refractory Mycoplasma pneumoniae in children (China). China Foreign Med Treat 2017; 35:132-5.
9. Hou J, Feng RS. Efficacy comparison of different doses of methylprednisolone combined with azithroycin in the treatment of children with refractory Mycoplasma pneumoniaa pneumonia. Int Med Health Guid News 2020; 26(23): 3606-8 doi: 10.3760/cma.j.issn.1007-1245.2020. 23.016 .

10. Liang R. Clinical study of methylprednisolone combined with azithromycin in the treatment of refractory mycoplasma pneumonia in children. Lab Med 2016; 31:293-4.

11. Zhou XM, Wei N. Efficacy and safety of methylprednisolone combined with azithromycin in the treatment of refractory mycoplasma pneumonia in children. Chin community doct 2018; 34(33):74-5.

12. Munakata M. Exhaled nitric oxide (FeNO) as a nonInvasive marker of airway inflammation. Allergol Int 2012; 3(61):365-72.doi:10.2332/allergolint.12-RAI-0461.

13. Sunadome $H$, Matsumoto $H$, Izuhara $Y$, Nagasaki $T$, Kanemitsu $Y$, Ishiyama $Y$, et al. Correlation between eosinophil count, its genetic background and body mass index: The nagahama study. Allergol Int 2020; 69(1): 46-52. doi:10.1016/j.alit.2019.05.012.

14. Zhang $Y$, Liu C. Clinical significance of FeNO and peripheral blood eosinophil ratio in children with Mycoplasma pneumoniae pneumonia. J Tianjin Med Univ 2019; 25(2): 147-9.

15. Xing H, Liu X, Li A, Li C, Wang J. Increased Peripheral TFH Cells and Serum Interleukin-21 in Childhood Mycoplasma Pneumoniae Pneumonia. J Coll Physicians Surg Pak 2021; 31(05):605-7. doi: 10.29271/jcpsp.2021.05.605

16. Sun $X$, Yan $H$. Effects of Azithromycin combined with Methylprednisolone in treatment of children with refractory Mycoplasmal pneumonia. Med J Chin People Health 2020; 32(4):39-41. doi:10.3969/j.issn. 1672-0369. 2020.04.016.

17. Gao J, Wu F. Association between fractional exhaled nitric oxide, sputum induction and peripheral blood eosinophil in uncontrolled asthma. Allergy Asthma Clin Immunol 2018; 14:21-30.doi:10.1186/s13223-018-0248-7.

18. Jin H , Zhan L, Mei S, Shao Z. Serum cytokines and FeNO in school-aged children with Mycoplasma pneumoniae pneumonia. Med Sci Monit 2020; 26:e923449-1-8.doi: 10.12659/MSM.923449. 